PROCEEDINGS OF THE

AMERICAN MATHEMATICAL SOCIETY

Volume 138, Number 5, May 2010, Pages 1711-1720

S 0002-9939(09)10174-0

Article electronically published on December 22, 2009

\title{
BROWNIAN MOTION IN A BALL IN THE PRESENCE OF SPHERICAL OBSTACLES
}

\author{
JULIE O'DONOVAN \\ (Communicated by Michael T. Lacey)
}

\begin{abstract}
We study the problem of when a Brownian motion in the unit ball has a positive probability of avoiding a countable collection of spherical obstacles. We give a necessary and sufficient integral condition for a regularly spaced collection to be avoidable.
\end{abstract}

\section{INTRODUCTION}

The setting in this paper is the unit ball, $\mathbb{B}=\left\{x \in \mathbb{R}^{d}:|x|<1\right\}$, in Euclidean space $\mathbb{R}^{d}$ where $d \geq 3$. We study the problem of when Brownian motion in the ball has a positive probability of avoiding a countable collection of spherical obstacles and thereby reaching the outer boundary of $\mathbb{B}$.

We denote by $\Lambda$ a sequence of points in $\mathbb{B}$. To each point $\lambda$ in this sequence we associate a spherical obstacle, $B\left(\lambda, r_{\lambda}\right)$, where

$$
B\left(\lambda, r_{\lambda}\right)=\left\{x:|\lambda-x| \leq r_{\lambda}\right\},
$$

and we denote by $\partial B\left(\lambda, r_{\lambda}\right)$ the boundary of this obstacle. We let $\mathcal{B}$ denote the countable collection of closed spherical obstacles,

$$
\mathcal{B}=\bigcup_{\lambda \in \Lambda} B\left(\lambda, r_{\lambda}\right)
$$

We assume that the spherical obstacles are pairwise disjoint and lie inside the ball $\mathbb{B}$ and that the origin lies outside $\mathcal{B}$. We call a collection of spherical obstacles avoidable if there is a positive probability that the Brownian motion, starting from the origin, hits the boundary of $\mathbb{B}$ before hitting any of the spherical obstacles in $\mathbb{B}$. This is equivalent to the positive harmonic measure at 0 of the boundary of the unit ball with respect to the domain $\Omega=\mathbb{B} \backslash \mathcal{B}$, consisting of the unit ball minus the obstacles, that is, $\omega(0, \partial \mathbb{B} ; \Omega)>0$.

In the setting of the unit disk, Ortega-Cerdà and Seip [7] gave an integral condition for a collection of disks to be avoidable. In 4, Carroll and Ortega-Cerdà gave an integral criterion for a configuration of balls in $\mathbb{R}^{d}, d \geq 3$, to be avoidable. Thus, it seems natural to ask if Ortega-Cerdà and Seip's result for the disk in the plane can be extended to the ball in space. A solution to this problem is the main result of this paper.

Received by the editors June 19, 2009, and, in revised form, August 24, 2009.

2010 Mathematics Subject Classification. Primary 31B05, 60J65.

Key words and phrases. Brownian motion, harmonic measure.

(C)2009 American Mathematical Society Reverts to public domain 28 years from publication 
Next, we put some restrictions on the distribution of the spherical obstacles. A collection of obstacles, $\mathcal{B}$, is regularly spaced if it is separated, in that there exists $\epsilon>0$ such that given any $\lambda, \lambda^{\prime} \in \Lambda$ with $|\lambda| \geq\left|\lambda^{\prime}\right|$, then $\left|\lambda-\lambda^{\prime}\right|>\epsilon(1-|\lambda|)$; uniformly dense, in that there exists $R$ with $0<R<1$ such that for $x \in \mathbb{B}$, the ball $B(x, R(1-|x|))$ contains at least one $\lambda \in \Lambda$; and finally, the radius $r_{\lambda}=\phi(|\lambda|)$, where $\phi:[0,1) \rightarrow[0,1)$ is a decreasing function.

Answering a question of Akeroyd in [2], Ortega-Cerdà and Seip [7] proved the following theorem.

Theorem A. A collection of regularly spaced disks in the unit disk is avoidable if and only if

$$
\int_{0}^{1} \frac{d t}{(1-t) \log ((1-t) / \phi(t))}<\infty
$$

This theorem in 7 is expressed in terms of pseudo-hyperbolic disks. We extend Theorem $\mathrm{A}$ to the setting of the unit ball in $\mathbb{R}^{d}, d \geq 3$.

Theorem 1.1. The collection of regularly spaced closed spherical obstacles $\mathcal{B}$ in $\mathbb{B}$ is avoidable if and only if

$$
\int_{0}^{1} \frac{\phi(t)^{d-2}}{(1-t)^{d-1}} d t<\infty
$$

We present two proofs of this result. The first proof exploits a connection between avoidability and minimal thinness, a potential theoretic measure of the size of a set near a boundary point of a region. We learned of this connection from both the paper of Lundh [6] and from Professor S.J. Gardiner. We also make use of a Wiener-type criterion for minimal thinness due to Aikawa [1].

The second proof is more direct and transparent. It is an adaptation of OrtegaCerdà and Seip's proof of Theorem $\mathrm{A}$ in [7, the key difference being that in higher dimensions we do not have the luxury of conformal mapping.

\section{Avoidable obstacles and minimal thinNess}

Following the notation of Lundh [6], we let $S H(\mathbb{B})$ denote the class of nonnegative superharmonic functions on the unit ball and let $P_{\tau}$ denote the Poisson kernel at $\tau \in \partial \mathbb{B}$. For a positive superharmonic function $h$ on $\mathbb{B}$ the reduced function of $h$ with respect to a subset $E$ of $\mathbb{B}$ is

$$
R_{h}^{E}(w)=\inf \{u(w): u \in S H(\mathbb{B}), u(x) \geq h(x), x \in E\}
$$

and the regularized reduced function is $\widehat{R}_{h}^{E}(w)=\liminf _{x \rightarrow w} R_{h}^{E}(x)$.

Definition 2.1. A set $E$ is minimally thin at $\tau \in \partial \mathbb{B}$ if there is an $x_{0}$ in the unit ball such that $\widehat{R}_{P_{\tau}}^{E}\left(x_{0}\right)<P_{\tau}\left(x_{0}\right)$.

A nice account of reduced functions and minimal thinness may be found in [5. pp. $38 \mathrm{ff}]$ or [3, Chapter 9].

2.1. Avoidability and minimal thinness. Lundh proves the following result in [6. We include a brief proof for the convenience of the reader.

Proposition 2.2. Let $A$ be a closed subset of $\mathbb{B}$ such that $\mathbb{B} \backslash A$ contains the origin and is connected. Let $\mathcal{M}=\{\tau \in \partial \mathbb{B}: A$ is minimally thin at $\tau\}$. Then the following are equivalent: 
- $A$ is avoidable,

- $|\mathcal{M}|>0$,

where |.| denotes surface area on the unit ball.

Proof. Noting that

$$
1=\int_{\partial \mathbb{B}} P_{\tau}(x) \frac{d \tau}{|\partial \mathbb{B}|}
$$

and taking $h \equiv 1$ in [3, Corollary 9.1.4], we see that

$$
\widehat{R}_{1}^{A}(x)=\int_{\partial \mathbb{B}} \widehat{R}_{P_{\tau}}^{A}(x) \frac{d \tau}{|\partial \mathbb{B}|} .
$$

Also, it follows from [5. p. $653,14.3 \mathrm{sm}$ ] that the regularized reduced function of 1 with respect to $A$ evaluated at $x$ is the harmonic measure at $x$ of $\partial A$ in the domain $\mathbb{B} \backslash A$. Thus,

$$
\omega(0, \partial A, \mathbb{B} \backslash A)=\widehat{R}_{1}^{A}(0)=\frac{1}{|\partial \mathbb{B}|} \int_{\partial \mathbb{B}} \widehat{R}_{P_{\tau}}^{A}(0) d \tau .
$$

Since $\widehat{R}_{P_{\tau}}^{A}(0) \leq P_{\tau}(0)=1$, it follows that $\omega(0, \partial A, \mathbb{B} \backslash A)<1$ if and only if the set $\mathcal{M}_{0}=\left\{\tau \in \partial \mathbb{B}, \widehat{R}_{P_{\tau}}^{A}(0)<1\right\}$ has positive measure. In the connected domain $\mathbb{B} \backslash A$, the set $\mathcal{M}_{0}$ is the same as the set $\mathcal{M}$. Thus, $A$ being avoidable, that is, $\omega(0, \partial \mathbb{B} ; \mathbb{B} \backslash A)>0$, is equivalent to $\mathcal{M}$ having positive measure.

2.2. Minimal thinness and a Wiener-type criterion. It is a standard result (see for example Aikawa [1] or Lundh [6]) that a set is minimally thin at a point if and only if it satisfies a Wiener-type criterion. Let $\left\{Q_{k}\right\}$ be a Whitney decomposition of the unit ball $\mathbb{B}$ in $\mathbb{R}^{d}(d \geq 3)$ and let $q_{k}$ be the Euclidean distance from the centre, $c_{k}$, of the Whitney cube $Q_{k}$ to the boundary of $\mathbb{B}$. Let $A$ be a subset of $\mathbb{B}$. Let $\tau$ be a boundary point of $\mathbb{B}$ and $\rho_{k}(\tau)$ be the distance from $c_{k}$ to the boundary point $\tau$. Let cap denote Newtonian capacity. Then $A$ is minimally thin at the point $\tau$ if and only if

$$
\sum_{k} \frac{q_{k}^{2}}{\rho_{k}(\tau)^{d}} \operatorname{cap}\left(A \cap Q_{k}\right)<\infty .
$$

In the next section, we consider this Wiener-type criterion in the particular setting of the unit ball minus a collection of regularly spaced spherical obstacles.

2.3. A Wiener-type criterion and an integral condition. For a constant $K>$ 1 , we let $S_{j}=\left\{x:|x|=1-K^{-j}\right\}$ be the sphere of radius $\beta_{j}=1-K^{-j}$ and $B_{j}$ be the interior of this sphere. We denote by $A_{j}$ the annulus bounded by $S_{j}$ and $S_{j-1}$, and we write $\phi_{j}$ for $\phi\left(\beta_{j}\right)$.

Proposition 2.3. Let $\mathcal{B}$ be a regularly spaced collection of spherical obstacles in $\mathbb{B}$.

(i) If the set $\mathcal{B}$ satisfies the Wiener-type criterion (2.1) at some point in $\partial \mathbb{B}$, then the integral condition (1.1) holds,

(ii) The integral condition (1.1) implies that $\mathcal{B}$ satisfies the Wiener-type criterion (2.1) at all points $\tau \in \partial \mathbb{B}$. 
Proof. We first assume that the integral condition holds and we will show that (2.1) follows. We note that the integral condition (1.1) is equivalent to

$$
\sum_{j=1}^{\infty}\left(\phi_{j} K^{j}\right)^{d-2}<\infty
$$

where $K>1$. By the separation condition on the sequence $\Lambda$, there is an $N$ such that any cube $Q_{k}$ can contain no more than $N$ points in $\Lambda$. Splitting the sum in (2.1) into a sum over annuli, we obtain

$$
\begin{aligned}
\sum_{k} \frac{q_{k}^{2}}{\rho_{k}(\tau)^{d}} \operatorname{cap}\left(\mathcal{B} \cap Q_{k}\right) & =\sum_{j=1}^{\infty} \sum_{k: c_{k} \in A_{j}} \frac{q_{k}^{2}}{\rho_{k}(\tau)^{d}} \operatorname{cap}\left(\mathcal{B} \cap Q_{k}\right) \\
& \leq \sum_{j=1}^{\infty} N\left(K^{-j}\right)^{2} \phi_{j}^{d-2} \sum_{k: c_{k} \in A_{j}} \frac{1}{\rho_{k}(\tau)^{d}}
\end{aligned}
$$

since the capacity of a ball with radius $\phi_{j}$ is equal to $\phi_{j}^{d-2}$. We now concentrate on the latter sum in (2.4). We split up the $j^{t h}$ annulus $A_{j}$ into rings centred at the projection of $\tau$ onto the sphere $S_{j}$, and with radius equal to $n K^{-j}$, where we recall that $K^{-j}$ is the distance from $\tau$ to $S_{j}$. There are at most

$$
\frac{c_{d}\left(n K^{-j}\right)^{d-2}}{\left(K^{-j}\right)^{d-2}}=c_{d} n^{d-2}
$$

Whitney cubes in each ring, where $c_{d}$ is a constant depending on the dimension, $d$. For the $n^{\text {th }}$ ring,

$$
\rho_{k}(\tau) \geq n K^{-j}
$$

and $N_{j}$ rings intersect the annulus $A_{j}$. Thus,

$$
\begin{aligned}
\sum_{k: c_{k} \in A_{j}} \frac{1}{\rho_{k}(\tau)^{d}} & \leq \sum_{n=1}^{N_{j}} \frac{c_{d} n^{d-2}}{\left(n K^{-j}\right)^{d}} \\
& \leq\left(K^{j}\right)^{d} c_{d} \sum_{n=1}^{N_{j}} \frac{1}{n^{2}} .
\end{aligned}
$$

Thus, we see that the Wiener-type series (2.3) is convergent.

We now assume that the set $\mathcal{B}$ satisfies (2.1) at some arbitrary point $\tau \in \partial \mathbb{B}$ and show that this implies the integral condition (1.1). We choose $K$ sufficiently large so that for all $j$ larger than a fixed constant there is at least one centre of a ball in each Whitney cube, $Q_{k}$, in the resulting Whitney decomposition of $\mathbb{B}$. Starting with the Wiener-type series we split it into a sum over the annuli $A_{j}$ and 
then proceed to ignore all Whitney cubes in $A_{j}$ except one near to the point $\tau$, for which $\rho_{k}(\tau) \leq K^{-j}$, as follows:

$$
\begin{aligned}
\sum_{k} \frac{q_{k}^{2}}{\rho_{k}(\tau)^{d}} \operatorname{cap}\left(\mathcal{B} \cap Q_{k}\right) & =\sum_{j=1}^{\infty} \sum_{k: c_{k} \in A_{j}} \frac{q_{k}^{2}}{\rho_{k}(\tau)^{d}} \operatorname{cap}\left(\mathcal{B} \cap Q_{k}\right) \\
& \geq \sum_{j=0}^{\infty} K^{-2 j} \phi_{j}^{d-2} \frac{1}{\rho_{k}(\tau)^{d}} \\
& \geq \sum_{j=0}^{\infty}\left(\phi_{j} K^{j}\right)^{d-2}
\end{aligned}
$$

Thus, since the Wiener-type series is convergent, (2.2) follows and so the integral condition (1.1) holds.

Combining Proposition 2.2, the Wiener-type criterion (2.1) and Proposition 2.3. we have a proof of Theorem 1.1. We note that the method used in this section could also be used to give an alternative proof of Ortega-Cerdà and Seip's Theorem A.

\section{Direct proof of Theorem 1.1}

We now give an alternative proof of Theorem 1.1 by adapting the method of Ortega-Cerdà and Seip in [7]. In dimensions higher than 2 we do not have conformal mappings, but we do have the Kelvin transform. We let

$$
x^{*}=\frac{\beta_{j+1}^{2}}{|x|^{2}} x
$$

be the inversion of the point $x$ in the sphere of radius $\beta_{j+1}$. We note that $|x|\left|x^{*}\right|$ equals $\beta_{j+1}^{2}$, and we let $\phi(|\lambda|)=\phi_{\lambda}$. We begin with some lemmas and prove the sufficiency of the integral condition in the next subsection and the necessity in the following one.

Lemma 3.1. Let $K>\max \left\{4, \frac{1+R}{1-R}\right\}$ and $x$ be an arbitrary point belonging to $S_{j-1}$. There exists a centre of an obstacle, $\lambda_{x} \in \Lambda$, such that $\lambda_{x}$ lies in the annulus $A_{j}$ bounded by $S_{j-1}$ and $S_{j}$ and

$$
\left|x-\lambda_{x}\right| \leq \frac{K-1}{K}\left|x^{*}-\lambda_{x}\right|
$$

Proof. For $x \in S_{j-1}$, let $x^{\prime}$ be the point on the extension of the radius of $S_{j}$ containing $x$ and located halfway between $S_{j-1}$ and $S_{j}$. Then $x^{\prime}$ is a distance $K^{-(j-1)}-\frac{K-1}{2 K^{j}}$ from the boundary of the ball $\mathbb{B}$. Since $\Lambda$ is uniformly dense, the ball $B\left(x^{\prime}, R\left(1-\left|x^{\prime}\right|\right)\right)$ contains some $\lambda_{x} \in \Lambda$. Also, due to the choice of $K$, the ball $B\left(x^{\prime}, R\left(1-\left|x^{\prime}\right|\right)\right)$ is contained in the annulus $A_{j}$. Let $x^{\prime \prime}$ be on the same ray as $x$ and $x^{*}$ and also on $S_{j}$. We first note that $\left|x-\lambda_{x}\right| \leq\left|x-x^{\prime \prime}\right|$ and $\left|x^{*}-\lambda_{x}\right|>\left|x^{*}-x^{\prime \prime}\right|$. Also, we note that $|x|=\beta_{j-1},\left|x^{\prime \prime}\right|=\beta_{j}$ and $\left|x^{*}\right|=\beta_{j+1}^{2} / \beta_{j-1}$. Thus,

$$
\left|x-\lambda_{x}\right| \leq\left|x-x^{\prime \prime}\right|=(K-1) K^{-j} .
$$

Also,

$$
\left|x^{*}-\lambda_{x}\right| \geq\left|x^{*}-x^{\prime \prime}\right|=\frac{\left(1-K^{-(j+1)}\right)^{2}}{1-K^{-(j-1)}}-\left(1-K^{-j}\right) \geq K^{-j+1},
$$


for $j \geq 2$. Thus,

as required.

$$
\left|x-\lambda_{x}\right| \leq \frac{K-1}{K}\left|x^{*}-\lambda_{x}\right|,
$$

The following lemma gives us an upper and a lower estimate for the harmonic measure of a spherical obstacle with respect to a ball minus that obstacle.

Lemma 3.2. For $\lambda \in A_{j},|\lambda|$ sufficiently close to 1 and $B_{\lambda} \subset \mathbb{B}$, the following estimates for the harmonic measure hold:

(i) If (1.1) holds, then $\omega\left(x, \partial B_{\lambda} ; \mathbb{B} \backslash B_{\lambda}\right) \leq h(x)$, where

$$
h(x)=2\left(\left[\frac{\phi_{\lambda}}{|x-\lambda|}\right]^{d-2}-\left[\frac{\phi_{\lambda}}{|x|\left|x^{*}-\lambda\right|}\right]^{d-2}\right) \text { and } x^{*}=\frac{1}{|x|^{2}} x ;
$$

(ii) $\omega\left(x, \partial B_{\lambda} ; B_{j+1} \backslash B_{\lambda}\right) \geq h_{j}(x)$, where

$$
h_{j}(x)=\left[\frac{\phi_{\lambda}}{|x-\lambda|}\right]^{d-2}-\left(\frac{\beta_{j+1}}{|x|}\right)^{d-2}\left[\frac{\phi_{\lambda}}{\left|x^{*}-\lambda\right|}\right]^{d-2} \text { and } x^{*}=\frac{\beta_{j+1}^{2}}{|x|^{2}} x .
$$

Proof. (i) We construct a suitable function $h$ that is harmonic on $\mathbb{B} \backslash B_{\lambda}$, continuous on its closure and also satisfies $h(x) \geq 1, x \in \partial B_{\lambda}$ and $h(x) \geq 0, x \in \partial \mathbb{B}$. Then, using the Maximum Principle, we obtain the required upper bound. Consider the function

$$
h(x)=2\left[u_{\lambda}(x)-u_{\lambda}^{*}(x)\right],
$$

where

$$
u_{\lambda}(x)=\left[\frac{\phi_{\lambda}}{|x-\lambda|}\right]^{d-2}, u_{\lambda}^{*}(x)=\left[\frac{\phi_{\lambda}}{|x|\left|x^{*}-\lambda\right|}\right]^{d-2} \text { and } x^{*}=\frac{1}{|x|^{2}} x .
$$

We note that $u_{\lambda}$ and $u_{\lambda}^{*}$ are harmonic and that they agree on $\partial \mathbb{B}$. Also, $1 / 2$ is a lower bound for $u_{\lambda}(x)-u_{\lambda}^{*}(x)$ for $x \in \partial B_{\lambda}$, which we show as follows. For $x \in \partial B_{\lambda}$, we have that $|x| \geq 1-K^{-1}$ and $\left|x^{*}-\lambda\right| \geq K^{-j}$; hence

$$
u_{\lambda}(x)-u_{\lambda}^{*}(x)=1-\left[\frac{\phi_{\lambda}}{|x|\left|x^{*}-\lambda\right|}\right]^{d-2} \geq 1-\left[\frac{K \phi_{j-1}}{(K-1) K^{-j}}\right]^{d-2} .
$$

It follows from (2.2) that

$$
\lim _{j \rightarrow \infty} \frac{\phi_{j-1}}{K^{-j}}=0 .
$$

Thus, there exists $N$ such that for $j>N$,

$$
u_{\lambda}(x)-u_{\lambda}^{*}(x)>\frac{1}{2} .
$$

Hence, $h(x)$ satisfies the required criteria and is an upper bound for $\omega\left(x, \partial B_{\lambda} ; \mathbb{B} \backslash B_{\lambda}\right)$.

(ii) We need a lower bound for $\omega\left(x, \partial B_{\lambda} ; B_{j+1} \backslash B_{\lambda}\right)$. We want a suitable function $h_{j}$ that is harmonic on $B_{j+1} \backslash B_{\lambda}$, continuous on its closure and also satisfies $h_{j}(x) \leq$ $1, x \in \partial B_{\lambda}$ and $h_{j}(x) \leq 0, x \in S_{j+1}$. Then we can again avail ourselves of the Maximum Principle to obtain the required lower bound. Consider the function

$$
h_{j}(x)=v_{\lambda}(x)-v_{\lambda}^{*}(x),
$$

where

$$
v_{\lambda}(x)=\left[\frac{\phi_{\lambda}}{|x-\lambda|}\right]^{d-2}, v_{\lambda}^{*}(x)=\left(\frac{\beta_{j+1}}{|x|}\right)^{d-2}\left[\frac{\phi_{\lambda}}{\left|x^{*}-\lambda\right|}\right]^{d-2} \text { and } x^{*}=\frac{\beta_{j+1}^{2}}{|x|^{2}} x .
$$


Then $h_{j}(x)$ satisfies the required criteria as both $v_{\lambda}$ and $v_{\lambda}^{*}$ are harmonic, $h_{j} \leq$ $v_{\lambda}=1$ on $\partial B_{\lambda}$, and $v_{\lambda}=v_{\lambda}^{*}$ on $S_{j+1}$.

We note that the functions $h$ and $h_{j}$ in the previous lemma are a suitable constant times the Green function for a ball.

3.1. Integral condition (1.1) implies avoidability. We first assume (1.1) and show that the spherical obstacles are avoidable; that is, we show that $\omega(0, \partial \mathbb{B} ; \Omega)>$ 0 . We split the collection of spherical obstacles into those with centres inside and those with centres outside a ball of radius $r<1$. We let $\Lambda_{r}=\{\lambda \in \Lambda:|\lambda|>r\}$ and let

$$
\mathcal{B}_{r}=\bigcup_{\lambda \in \Lambda_{r}} B\left(\lambda, r_{\lambda}\right)=\bigcup_{\lambda \in \Lambda_{r}} B_{\lambda}
$$

denote the infinitely many spherical obstacles with centres outside $B(0, r)$. Also, we let $\Omega_{r}=\mathbb{B} \backslash \mathcal{B}_{r}$ be the champagne subregion where all obstacles have centres outside a ball of radius $r$. We may safely ignore the finitely many spherical obstacles with centres inside the ball of radius $r$. Thus, it is sufficient to show that $\omega\left(0, \partial \mathbb{B} ; \Omega_{r}\right)>0$ for some $r$ with $0<r<1$, which is equivalent to showing that $\omega\left(0, \partial \mathcal{B}_{r} ; \Omega_{r}\right)<1$. We choose $r$ such that

$$
\int_{r}^{1} \frac{\phi(t)^{d-2}}{(1-t)^{d-1}} d t<\frac{\epsilon^{d}(K-1)^{d-2}}{2^{d+1} d(d-2) K^{2 d-1}}
$$

and let $n_{r}$ be the biggest integer smaller than $1+\log \left(\frac{1}{1-r}\right) / \log K$. This ensures that $r>\left\lfloor 1-K^{-\left(n_{r}-1\right)}\right\rfloor$. We proceed as follows:

$$
\begin{aligned}
\omega\left(0, \partial \mathcal{B}_{r} ; \Omega_{r}\right)=\sum_{\lambda \in \Lambda_{r}} \omega\left(0, \partial B_{\lambda} ; \Omega_{r}\right) & \leq \sum_{\lambda \in \Lambda_{r}} \omega\left(0, \partial B_{\lambda} ; \mathbb{B} \backslash B_{\lambda}\right) \\
& \leq \sum_{j=n_{r}}^{\infty}\left(\sum_{\lambda \in A_{j}} \omega\left(0, \partial B_{\lambda} ; \mathbb{B} \backslash B_{\lambda}\right)\right) .
\end{aligned}
$$

We now obtain an upper bound for the number of centres in $A_{j}$ and an upper bound for the contribution of an obstacle with centre in $A_{j}$ to the above sum. Due to the separation condition, centres of balls in $A_{j}$ are at least $\epsilon K^{-j}$ apart. Thus, the number of centres in $A_{j}$, which is less than the volume of $A_{j}$ divided by the volume of a ball with radius $\epsilon K^{-j} / 2$, is less than

$$
\frac{2^{d} d K^{2}}{\epsilon^{d}} K^{(d-1) j}
$$

We want an upper bound for $\omega\left(0, \partial B_{\lambda} ; \mathbb{B} \backslash B_{\lambda}\right)$. By Lemma 3.2 an upper bound for $\omega\left(x, \partial B_{\lambda} ; \mathbb{B} \backslash B_{\lambda}\right)$ is given by $h(x)$. Thus, we want an upper estimate for $h(0)$. We first note that as $x \rightarrow 0, x^{*} \rightarrow \infty$ and also that $|x|\left|x^{*}\right|=1$. Thus, as $x \rightarrow 0$, $u_{\lambda}^{*}(x) \rightarrow \phi_{\lambda}^{d-2}$. Next,

$$
\begin{aligned}
\frac{1}{2} h(0) & =\lim _{x \rightarrow 0}\left[u_{\lambda}(x)-u_{\lambda}^{*}(x)\right]=\left(\frac{\phi_{\lambda}}{|\lambda|}\right)^{d-2}-\phi_{\lambda}^{d-2}=\phi_{\lambda}^{d-2}\left[\frac{1-|\lambda|^{d-2}}{|\lambda|^{d-2}}\right] \\
& \leq\left(\frac{\phi_{j-1}}{|\lambda|}\right)^{d-2}(d-2)\left[K^{-(j-1)}+O\left(K^{-2 j}\right)\right] .
\end{aligned}
$$


Thus, for sufficiently large $j$,

$$
h(0) \leq 4 K(d-2)\left(\frac{\phi_{j-1}}{1-K^{-(j-1)}}\right)^{d-2} K^{-j} .
$$

Therefore,

$$
\begin{aligned}
\omega\left(0, \partial \mathcal{B}_{r} ; \Omega_{r}\right) & \leq \sum_{j=n_{r}}^{\infty} \frac{2^{d} d K^{2}}{\epsilon^{d}} K^{(d-1) j} 4 K(d-2)\left(\frac{\phi_{j-1}}{1-K^{-(j-1)}}\right)^{d-2} K^{-j} \\
& \leq \frac{2^{d+2} d(d-2) K^{2 d-1}}{\epsilon^{d}(K-1)^{d-2}} \sum_{j=n_{r}}^{\infty}\left(\phi_{j-1} K^{j-1}\right)^{d-2}<1
\end{aligned}
$$

provided $n_{r}$ is suitably selected as described at the start of the proof. Thus, $\omega\left(0, \partial \mathcal{B}_{r} ; \Omega_{r}\right)<1$, and hence we see that $\omega(0, \partial \mathbb{B} ; \Omega)>0$ as required.

3.2. Avoidability implies the integral condition (1.1). Now we assume that $\omega(0, \partial \mathbb{B} ; \Omega)>0$, and we will show that (1.1) holds. We begin by ignoring all obstacles with centres in an annulus $A_{j}$ where $j$ is odd. We let

$$
\Omega^{\prime}=\mathbb{B} \backslash \bigcup_{\lambda \in A_{j}, j \text { even }} B\left(\lambda, r_{\lambda}\right)
$$

and note that since $\omega(0, \partial \mathbb{B} ; \Omega)>0$, then $\omega\left(0, \partial \mathbb{B} ; \Omega^{\prime}\right)>0$. We choose $K>$ $\max \left\{4, \frac{1+R}{1-R}\right\}$, where $R$ is the constant mentioned in the definition of a regularly spaced collection of obstacles. For $j$ even, we let $P_{j}$ denote the probability that Brownian motion starting at the origin hits $S_{j+1}$ before hitting any of the obstacles with centres in $B_{j}$ but not in any $A_{i}$, where $i$ is odd. We let $Q_{j}$ denote the supremum of the probabilities that Brownian motion starting on $S_{j-1}$ hits $S_{j+1}$ before hitting any of the obstacles with centres in $A_{j}$. We note that $P_{j} \leq Q_{j} P_{j-2}$ and that therefore for $n$ even,

$$
P_{n} \leq P_{0} \prod_{j=1, j \text { even }}^{n} Q_{j}
$$

Since $\omega\left(0, \partial \mathbb{B} ; \Omega^{\prime}\right)=\delta>0$, it follows that $P_{n} \geq \delta$ for all $n$ and, since $Q_{j}<1$,

$$
\sum_{j=1, j \text { even }}^{\infty}\left(1-Q_{j}\right)<\infty .
$$

We note that $1-Q_{j}$ is the infimum over $x \in S_{j-1}$ of the probability that Brownian motion starting at $x$ hits a ball with centre in $A_{j}$ before hitting $S_{j+1}$. Thus, if we consider only a single ball near $x$, say $B_{\lambda_{x}}$, where $\lambda_{x}$ is the centre of the ball near $x$ as described in Lemma 3.1, then

$$
1-Q_{j} \geq \inf _{x \in S_{j-1}} \omega\left(x, \partial B_{\lambda_{x}} ; B_{j+1} \backslash B_{\lambda_{x}}\right) .
$$

Thus, we need a lower bound for $\omega\left(x, \partial B_{\lambda_{x}} ; B_{j+1} \backslash B_{\lambda_{x}}\right)$. We have from Lemma 3.2 that $\omega\left(y, \partial B_{\lambda_{x}} ; B_{j+1} \backslash B_{\lambda_{x}}\right) \geq h_{j}(y)$. Hence, we want a lower estimate for $h_{j}$ at the 
point $x \in S_{j-1}$. With the help of Lemma 3.1

$$
\begin{aligned}
h_{j}(x) & =\left[\frac{\phi_{\lambda}}{\left|x-\lambda_{x}\right|}\right]^{d-2}-\left(\frac{\beta_{j+1}}{\beta_{j-1}}\right)^{d-2}\left[\frac{\phi_{\lambda}}{\left|x^{*}-\lambda_{x}\right|}\right]^{d-2} \\
& \geq\left(\frac{\phi_{j}}{\left|x-\lambda_{x}\right|}\right)^{d-2}\left[1-\left(\frac{\beta_{j+1}}{D \beta_{j-1}}\right)^{d-2}\right],
\end{aligned}
$$

where $D=K /(K-1)>1$. Then for sufficiently large $j$, namely $j$ where

$$
\frac{\beta_{j+1}}{\beta_{j-1}}<\frac{1+D}{2}
$$

we find that

$$
h_{j}(x) \geq c\left(\frac{\phi_{j}}{\left|x-\lambda_{x}\right|}\right)^{d-2}
$$

where $c$ is some positive constant.

By (3.1), we find that for $x \in S_{j-1}$,

$$
\omega\left(x, \partial B_{\lambda_{x}} ; B_{j+1} \backslash B_{\lambda_{x}}\right) \geq h_{j}(x) \geq c(K-1)^{2-d}\left(\phi_{j} K^{j}\right)^{d-2} .
$$

It now follows from (3.2) that

$$
\sum_{j=1, j \text { even }}^{\infty}\left(\phi_{j} K^{j}\right)^{d-2}<\infty .
$$

Similarly it may be shown that

$$
\sum_{j=1, j \text { odd }}^{\infty}\left(\phi_{j} K^{j}\right)^{d-2}<\infty,
$$

and so

$$
\sum_{j=1}^{\infty}\left(\phi_{j} K^{j}\right)^{d-2}<\infty .
$$

Hence, (1.1) holds and the proof is complete.

\section{ACKNowledgements}

The author would like to thank Tom Carroll for his help and guidance throughout this work and the referee for useful comments and suggestions.

\section{REFERENCES}

[1] H. Aikawa, Thin sets at the boundary, Proc. London Math. Soc. 65(3) (1992), 357-382. MR1168192 (93g:31012)

[2] J. R. Akeroyd, Champagne subregions of the unit disk whose bubbles carry harmonic measure, Math. Ann. 323 (2002), 267-279. MR1913043(2003c:30020)

[3] D. H. Armitage, S. J. Gardiner, Classical Potential Theory, Springer, 2001. MR.1801253 (2001m:31001)

[4] T. Carroll, J. Ortega-Cerdà, Configurations of balls in Euclidean space that Brownian motion cannot avoid, Ann. Acad. Sci. Fenn. Math. 32 (2007), 223-234. MR2297888(2008a:60193)

[5] J. Doob, Classical Potential Theory and Its Probabilistic Counterpart, Springer, 1984. MR731258 (85k:31001) 
[6] T. Lundh, Percolation diffusion, Stochastic Process. Appl. 95 (2001), 235-244. MR 1854027 (2003a:82036)

[7] J. Ortega-Cerdà, K. Seip, Harmonic measure and uniform densities, Indiana Univ. Math. J. 53(3) (2004), 905-923. MR2086705 (2005f:30051)

Department of Mathematics, University College Cork, Cork, Ireland

E-mail address: j.odonovan@ucc.ie 